\title{
Dispositivo para medir tiempo y temperatura usando un microcontrolador
}

\author{
Device for measuring time and temperature using a microcontroller \\ Germán Calderón ${ }^{1}$, José Herman Muñoz ${ }^{* 2}$, Javier Yovany Rivera² \\ ${ }^{1}$ Facultad de Ingeniería Mecánica y Eléctrica, Universidad Autónoma de Coahuila, C.P. 27276 Torreón, Coahuila, México \\ ${ }^{2}$ Departamento de Física, Universidad Tolima, A. A.546, Ibagué, Colombia
}

Recibida en 23 de Julio, 2017. Revisado en 01 de Octubre, 2017. Aceptado en 10 de Octubre, 2017.

\begin{abstract}
En este trabajo se desarrolló un dispositivo de adquisición de datos, de bajo costo y fácil manejo, para usar en experimentos de física donde se requiera medir tiempo o temperatura, conformado por tres equipos principales: (i) Sensores para medir las variables físicas de tiempo y temperatura; (ii) Una tarjeta de adquisición con microcontrolador para el manejo de instrucciones y (iii) Una computadora para el almacenamiento de datos. El sensor, compuesto de un infrarrojo y un fototransistor, cambia su estado lógico al interrumpir la señal. La tarjeta que se utiliza incluye un microcontrolador de la marca Microchip, PIC18F14K50, el cual contiene comparadores, convertidores de análogo a digital, temporizadores y puerto de comunicación USB (Universal Serial Bus). El computador se usó como una herramienta de almacenamiento de datos y visualización de variables a través de una hoja de cálculo. La programación del microcontrolador se hizo con software MPLAB X de licencia gratuita. La funcionalidad del dispositivo se verificó en el experimento de caída libre para determinar el valor de la aceleración gravitacional.
\end{abstract}

Palabras clave: Sistema de adquisición de datos, Sensor, Microcontrolador.

In this paper a device of data acquisition with low cost and easy management was developed for using in experiments of physics where time or temperature measurement is required. The device is composed by three main parts: (i) sensors to measure the physical variables of time and temperature; (ii) a data acquisition card with microcontroller to manage commands and (iii) a computer in order to store data. The sensor, composed by an infrared and a phototransistor, changes its logical state when the signal is interrupted. The board used includes a microcontroller of Microchip brand, PIC18F14K50, which has comparators, analog to digital converters, timers and an Universal Serial Bus (USB) port. The computer was used as a tool to store data and see variables by means of a spreadsheet. The microcontroller programming was made with MPLABX software of free license. The usefulness of the device was tested in the free fall experiment in order to determine the value of the gravitational acceleration.

Keywords: Data acquisition system, Sensor, Microcontroller.

\section{Introducción}

El proceso de enseñanza-aprendizaje de las ciencias naturales requiere el uso de laboratorios que faciliten al estudiante asimilar y reforzar conceptos estudiados teóricamente. Sin embargo, una buena cantidad de instituciones educativas (de nivel secundario y universitario), debido a dificultades presupuestales, no pueden comprar equipos con tecnología de punta ya que son costosos y su mantenimiento es dispendioso. Es decir, no cuentan con los equipos requeridos para ofrecer este apoyo didáctico, limitando la formación de los estudiantes a formalismos teóricos, muchas veces descontextualizados, y generando una carencia fundamental en el aprendizaje de las ciencias naturales [1].

*Correo electrónico: jhmunoz@ut.edu.co
Con el objetivo de contribuir a la solución del problema mencionado, en este trabajo se describe la construcción de un Sistema de Adquisición de Datos (SAD), de bajo costo y fácil manejo, para medir las variables físicas tiempo o temperatura dependiendo del experimento donde se utilice, usando un microcontrolador PIC18F14K50 programado con MPLABX, el cual tiene licencia gratuita, y compilado en lenguaje C. Este dispositivo se utilizó en el experimento de caída libre para determinar el valor de la gravedad.

La implementación con enfoque pedagógico adecuado de las Tecnologías de la Información y la Comunicación (TIC), de bajo costo o gratuitas, en la enseñanza de las ciencias es una herramienta útil que permite mejorar el proceso de enseñanza-aprendizaje y la generación de un pensamiento crítico [1-3]. Actualmente, es posible hablar del uso de las TIC como una línea de investigación de 
la didáctica de las ciencias [4, 5]. Algunas modalidades dentro de este campo son la Modelación y Simulación Computacional (MSC) [4] y el uso de SAD [5].

En el área de la física, en la dirección de la MSC, se encuentran recursos tecnológicos gratuitos para apoyar los procesos de enseñanza-aprendizaje de esta disciplina. Actualmente, se dispone de smartphones con diferentes aplicaciones (Apps) (ver, por ejemplo, las referencias [613]), del uso de programas de análisis de video como el Tracker (algunas referencias recientes son [14-18]), de la simulación de experimentos como lo ofertado por PhET de la Universidad de Colorado [19], etc. Sin embargo, estos recursos didácticos no desarrollan las competencias que genera la construcción de prototipos como el elaborado en el presente trabajo.

Por otra parte, para diseñar y construir un SAD que mida observables físicas, es posible utilizar diversos dispositivos. Por ejemplo, la tarjeta Arduino con licencia de código abierto con variados sensores (ver, por ejemplo, las referencias [20-30]); los microcontroladores PIC16F628A [31], PIC16F877 [32, 33] y el PIC18F14K50 (utilizado en este trabajo); las fotocompuertas y tarjetas de sonido para la adquisición de datos (ver, entre otras, las referencias [34-44]), entre otros. La mayoría de estos dispositivos son de bajo costo y utilizan lenguajes de programación de uso gratuito. Estos SAD son herramientas didácticas adicionales que, con un enfoque pedagógico apropiado, facilitan el aprendizaje significativo a través de la integración de tópicos de física, electrónica y programación. Además, disminuyen el tiempo en la toma e interpretación de datos y eleva la precisión en la toma de los mismos.

En la literatura reciente se encuentran diversos SAD de bajo costo para medir intervalos de tiempo, a la escala de milisegundos, usando diferentes herramientas. Por ejemplo, en las referencias [20, 22, 25, 28, 30] utilizaron la tarjeta Arduino, en [31, 33] incluyeron microcontroladores PIC, en $[34,36,37]$ consideraron la tarjeta de sonido del computador, los autores de [38, 41-44] usaron fotocompuertas, en los trabajos [45, 46] emplearon una cámara fotográfica digital y en [47] se valieron de un sensor ultrasónico. Por otra parte, en cuanto a SAD para medir temperatura, la mayoría de trabajos recientes [21, 23, 26] usan la tarjeta Arduino con diversos sensores como LM35, DS18B20, DHT11 y TMP36. El SAD presentado en este artículo usa un microcontrolador PIC 18F14K50 y da la posibilidad de medir tiempo o temperatura, dependiendo del experimento en el que se use. Está inspirado, principalmente, en los trabajos [22, 28, $31,34,38]$ y puede considerarse como una actualización y generalización de la referencia [31].

El artículo está organizado de la siguiente manera: en la sección 2 se presenta el prototipo describiendo sus tres partes principales; la sección 3 explica la programación del microcontrolador mostrando el código del sensor de interrupciones para medir el tiempo o la temperatura; el uso del equipo en el experimento de caída libre para determinar el valor de la gravedad se explica en la sección 4. Finalmente, las conclusiones se presentan en la sección 5 .

\section{Descripción del prototipo desarrollado}

El dispositivo está compuesto por un equipo que contiene: una tarjeta de adquisición, una torre de aluminio de $1.20 \mathrm{~m}$ y una aplicación denominada "Aprende Física" desarrollada en MPLAB X, compilador XC8 y Visual Studio.

(i) La tarjeta de adquisición esta ensamblada en una caja plástica de $14 \mathrm{~cm}$ x $10 \mathrm{~cm}$ x $5 \mathrm{~cm}$ que contiene dos circuitos impresos (ver figura 1) con sus correspondientes elementos: un microcontrolador PIC18F14K50, un cristal externo, dos capacitores, un relevador, seis LED, once transistores, conector de nueve pines (DB-9) y puertos de entrada y salida.

Esta parte del equipo se encarga de comunicar el sensor de interrupciones con los puertos de entrada y salida del microcontrolador, quien a su vez es conectado con la computadora a través de un cable USB como se muestra en la figura 2.

El circuito impreso de la figura 2 se diseñó en ARES (herramienta para la elaboración de placas de circuito impreso). La parte (a) es la tarjeta principal donde se montó el PIC18F14K50, el relevador, los transistores, las resistencias, el cristal, los capacitores y los diodos LED. La parte (b) es la tarjeta que contiene los conectores para los sensores, la fuente de $20 \mathrm{~V}$ y el electroimán.

La tarjeta de adquisición también contiene un microcontrolador PIC18F14K50 de la marca MICROCHIP (ver figura 3). En la página web [48] se dispone de forma gratuita de la hoja de datos técnicos del microcontrolador usado, de la plataforma integrada de desarrollo MPLAB $\mathrm{X}$ [49] y del compilador XC8. Éste microcontrolador es la parte principal del dispositivo. La arquitectura del PIC 18 es la más desarrollada de los microcontroladores de la familia de 8 bits, tiene 20 pines, tres de ellos para interrupciones, rendimiento hasta de 16 MIPS, memoria

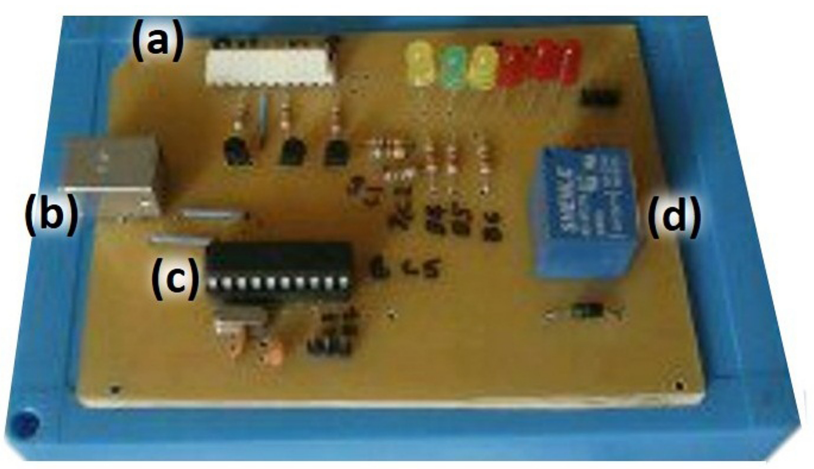

Figura 1: Tarjeta de adquisición con microcontrolador: (a) Puertos I/O; (b) Conexión USB; (c) Microcontrolador PIC 18F14K50; (d) Relay. 


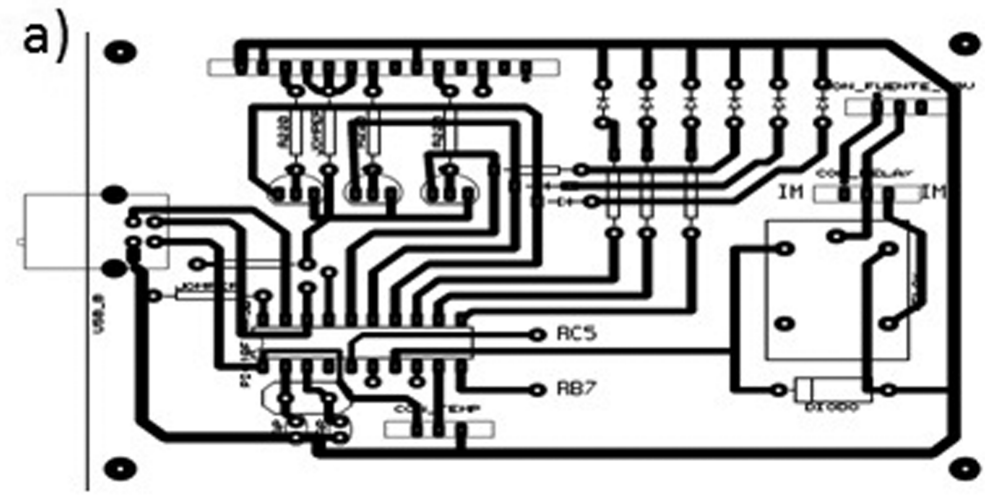

b)

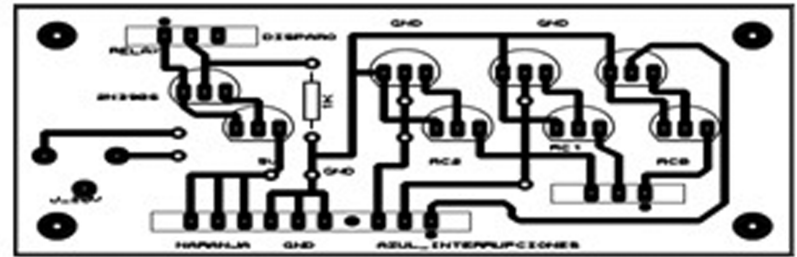

Figura 2: Circuito impreso: (a) tarjeta principal; (b) tarjeta de ajuste de altos y bajos ( 0 y 1$)$.

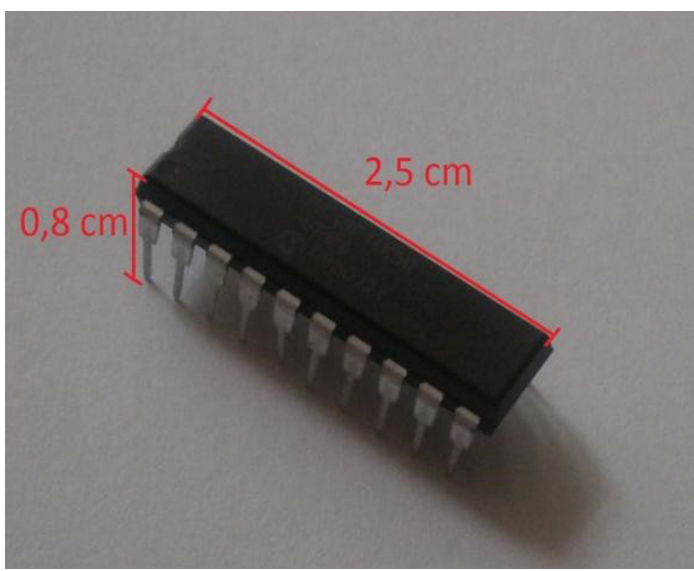

Figura 3: Microcontrolador PIC 18F14K50.

del programa hasta de $128 \mathrm{~KB}$, memoria de datos de 4 KB [50], optimizada para la programación en lenguaje C, de bajo costo. Adicionalmente, la tarjeta de adquisición contiene un conector de salida o entrada para medir las interrupciones y la temperatura. El relevador es para controlar el encendido o apagado del electroimán de la torre.

Otro componente de la tarjeta de adquisición es el conector de nueve pines (DB-9), el cual es una salida que va a la torre para alimentar el sensor de interrupciones. En la figura 4a se presenta el esquema del sensor de interrupción y en la figura 4b se muestra la estructura, en forma de herradura, para sostener en cada extremo el LED de infrarrojo (LED IR) y al fototransistor. a) CONECTOR A PUERTO DE INTERRUPCIONES

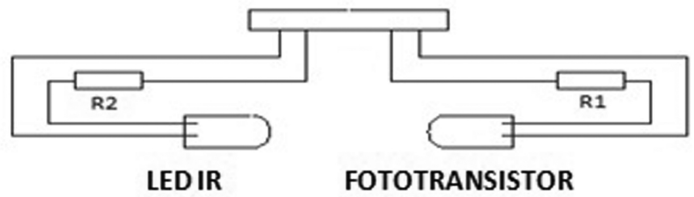

b)

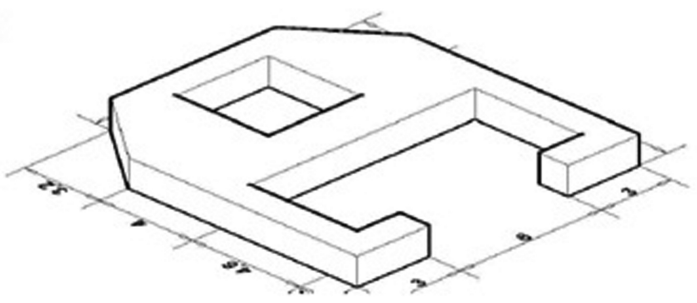

Figura 4: Esquema del sensor de interrupciones: (a) esquema eléctrico; (b) forma externa (Las distancias están dadas en $\mathrm{cm}$ ).

El sensor de interrupciones consta de un LED IR ubicado al frente de un fototransistor. Este LED IR emite constantemente luz de longitud de onda $900 \mathrm{~nm}$ que es recibida por el fototransistor; si la luz es interrumpida entonces el fototransistor deja de conducir corriente eléctrica y se produce un cambio de estado, de alto a bajo, el cual es usado como interrupción externa de hardware por el microcontrolador para medir diferencias de tiempo.

(ii) La torre (ver figura 5) consta de un tubo de aluminio y tres sensores de interrupciones acoplados a lo largo del tubo. Sus dimensiones son: $1.20 \mathrm{~m}$ x $0.03 \mathrm{~m}$ x $0.03 \mathrm{~m}$. 


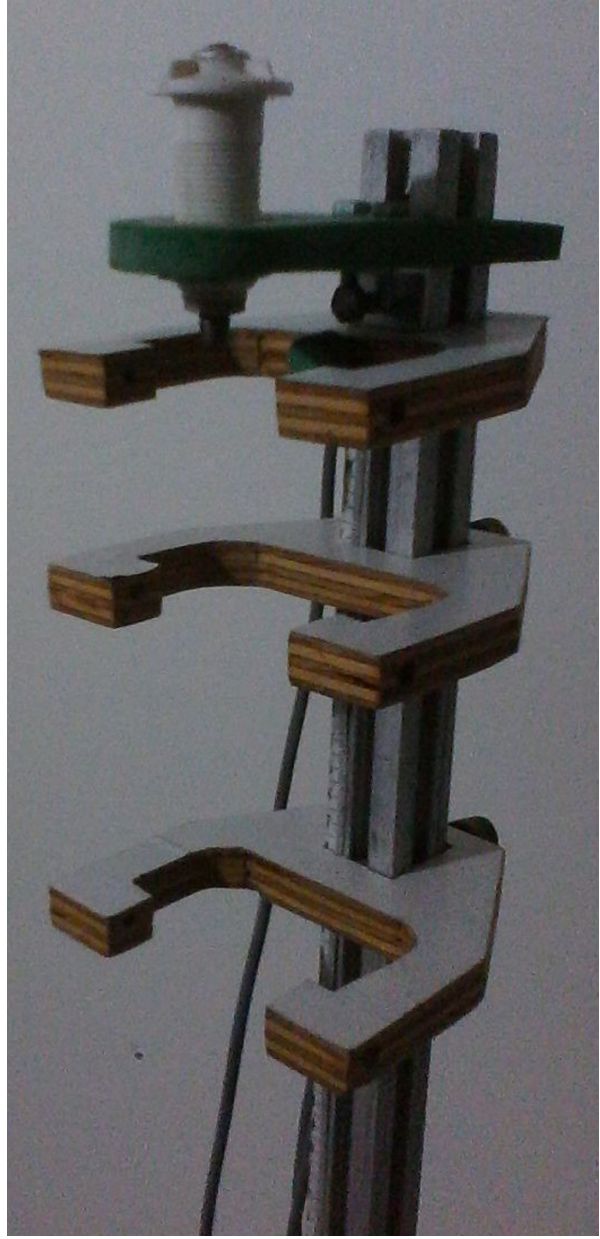

Figura 5: Torre.

A los sensores se les puede modificar su posición vertical, aflojando un tornillo en la parte posterior del sensor y llevándolo al lugar deseado. El tubo debe apoyarse en una base de madera nivelada, debiendo quedar vertical para su correcto uso.

La torre también contiene un electroimán, alimentado con una fuente de voltaje de $20 \mathrm{~V}$ de corriente directa y acoplado en el extremo superior del tubo (ver figura 5). Su función es sostener y soltar la esfera de acero que se usa en el experimento de caída libre. Mediante una instrucción de estado de la salida digital se controla el estado del electroimán como encendido o apagado, determinando el tiempo de inicio de la caída libre de la esfera.

Adicionalmente, se cuenta con el sensor LM35 para medir temperatura, de la marca Texas Instruments con las características descritas por el fabricante en [51]: $10 \mathrm{mV} /{ }^{\circ} \mathrm{C}$, rango de $-55^{\circ} \mathrm{C}$ a $150{ }^{\circ} \mathrm{C}$. El sensor LM35 tiene tres pines: uno se conecta a GND, el extremo izquierdo a $5 \mathrm{~V}$ y el del centro al microcontrolador.

(iii) Por último, la aplicación "Aprende Física" se desarrolló en Visual Studio, este es una interfaz de desarrollo gráfico con licencia de Microsoft. La aplicación permite la comunicación de la tarjeta de adquisición de datos con la computadora por medio del puerto USB, permitiendo la interacción del usuario con el prototipo desarrollado, dar inicio al experimento, almacenar la información y los resultados de los experimentos e interpretarlos.

La figura 6 muestra la aplicación "Aprende Física" una vez conectado a la tarjeta de adquisición de datos. En este se visualizan dos variables físicas: tiempo y temperatura; de igual manera presenta las ayudas para que el usuario las ejecute paso a paso.

\section{Programación del microcontrolador}

Para la programación del microcontrolador PIC18F14K50 se usó el software MPLAB X y el compilador XC8. El software MPLAB X, el cual es gratuito, proporcionado por la empresa Microchip Technology Inc, es un entorno de desarrollo integrado (IDE por sus siglas en inglés) similar a Microsoft Visual Studi, Codeblocks o NetBeans. Tiene todas las herramientas necesarias para escribir proyectos, editar archivos de extensión c, h y de assembler, compilar proyectos, simular en la computadora, depurar códigos, programar los microcontroladores, depurar en tiempo real, etc.

El Compilador $X C 8$ es un compilador diseñado para convertir el lenguaje $\mathrm{C}$ de programación al lenguaje de máquina, el cual decodifica y ejecuta el microcontrolador. El MPLAB X IDE se instala, por defecto, con el compilador del lenguaje ensamblador o assembler. Además, se pueden descargar otros compiladores como MPLAB XC8 (proporcionado por la Microchip Technology Inc) para programar en lenguaje $\mathrm{C}$ los microcontroladores.

Una vez creado el proyecto en MPLAB X se debe incluir en el editor de texto (archivo main.c) como mínimo las siguientes librerías: p18F14K50.h, stdio.h (entradas y salidas), stdlib.h, y la del compilador XC8. Adicionalmente, se configuran los pines del microcontrolador, como

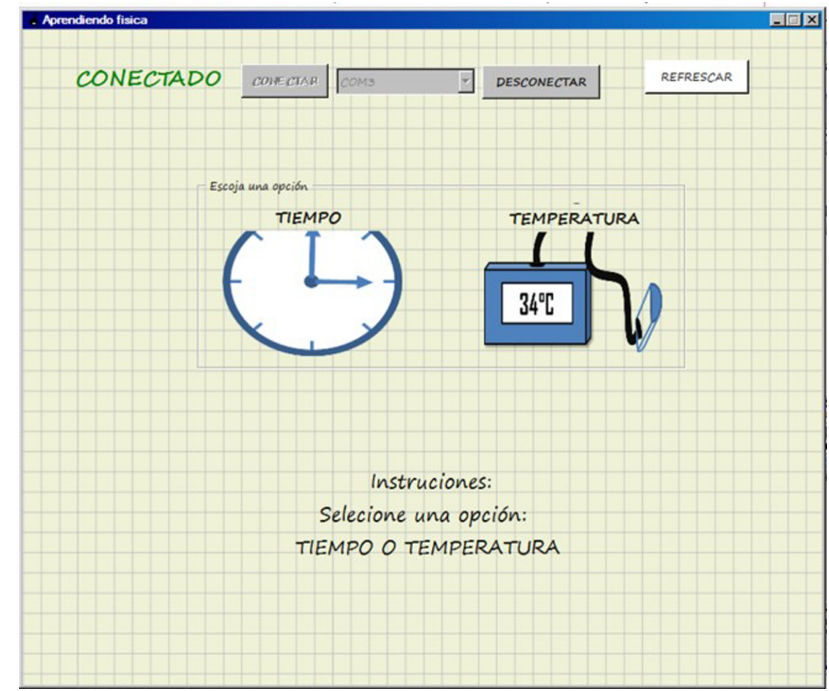

Figura 6: Aplicación "Aprende Física". 
puertos digitales, análogos, salidas y entradas, ver figura 7.

Para detectar el cambio de estado (por ejemplo el paso de una esfera en caída libre) a través del sensor que activa el reloj del microcontrolador PIC 18F14K50 fue necesario configurar el módulo de interrupciones con el siguiente procedimiento:

- Habilitar la interrupción que se va a utilizar.

- Habilitar las interrupciones globales.

- Definir si el flanco es ascendente o descendente.

- Definir la prioridad de cada interrupción (alta o baja).

- Borrar bandera de la interrupción.

El código escrito en lenguaje C, mostrado en la figura 8, presenta un ejemplo de interrupción por hardware externo en el pin $\mathrm{RC} 0$. Con un cambio de estado, de bajo a alto, el programa principal va a la rutina de interrupciones y luego va a la subrutina del temporizador, iniciando el conteo del tiempo con el temporizador [49], de este modo cuando la esfera pasa por el segundo sensor se obtiene el registro de su primer desplazamiento y cuando pasa por el tercer sensor registra el tiempo del recorrido mayor.

El dispositivo desarrollado también se puede utilizar para medir la temperatura. La figura 9 muestra el código en lenguaje $\mathrm{C}$ que permite dar inicio y esperar hasta que termina la conversión del Convertidor Análogo Digital (ADC). El ADC es un conversor de 10 bits de precisión,

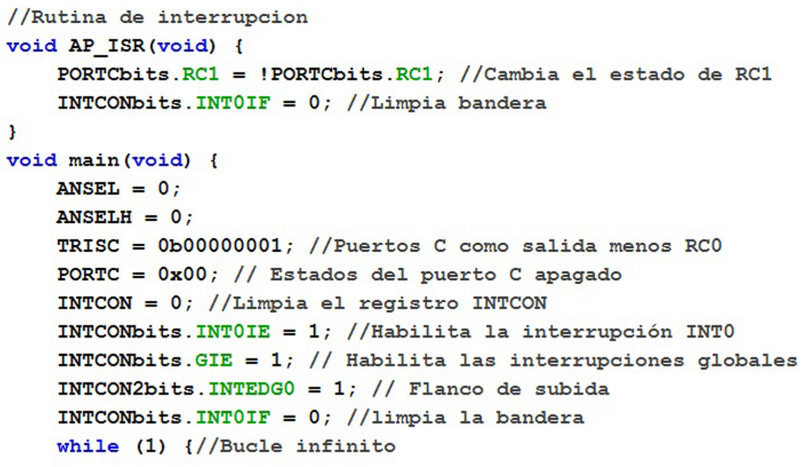

Figura 8: Código en lenguaje C para interrupciones.

que convierte lecturas de voltaje de 0 a $5 \mathrm{~V}$ a un número entero de 0 a 1023. Este número entero se escala para dar los valores apropiados en grados centígrados y así medir la temperatura usando el SAD en experimentos donde se requiera medir esta variable [52].

\section{Aplicaciones}

En esta sección se muestran los resultados obtenidos con el sistema de adquisición de datos desarrollado en este trabajo para medir el tiempo en el experimento de caída libre con el objetivo de obtener el valor de la gravedad.

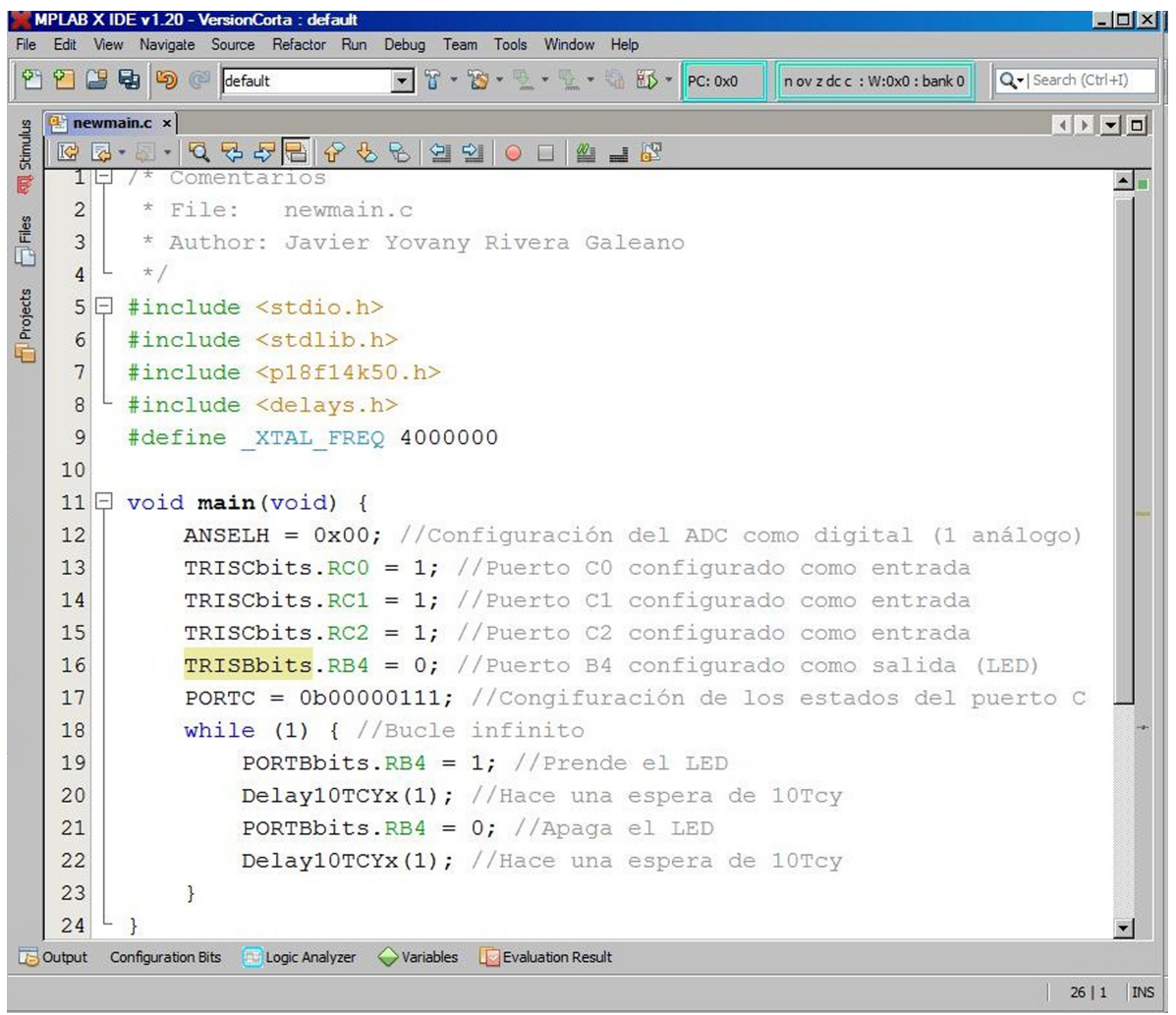

Figura 7: Código básico en MPLAB X. 


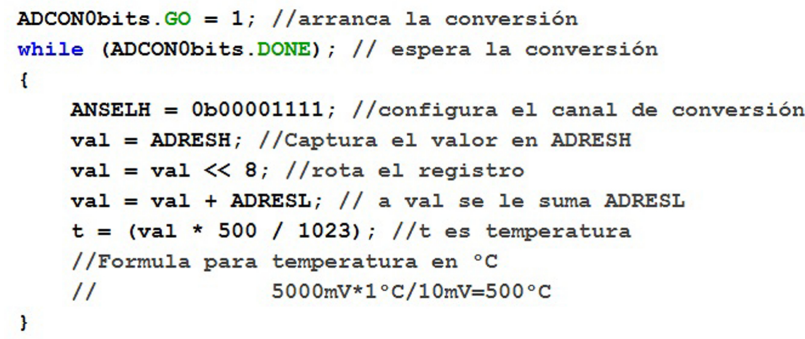

Figura 9: Código en lenguaje $C$ para medir la temperatura

Nuestros resultados se comparan con los obtenidos con el aparato de la marca LEYBOLD (ver figura 10).

La tabla 1 presenta los tiempos en segundos (s) y los desplazamientos en metros (m) para una esfera de acero, de radio $6.33 \mathrm{~mm}$, en caída libre. Teniendo en cuenta que la torre (ver figura 5) solo tiene tres sensores, dos de ellos se desplazaron a lo largo del tubo vertical para tomar los valores que se muestran en esta tabla. El experimento se repitió cinco veces para promediar los valores y luego graficarlos con el objetivo de obtener la aceleración gravitacional local.

La figura 11 presenta la gráfica del desplazamiento $\mathrm{S}(\mathrm{m})$ contra el tiempo $\mathrm{t}(\mathrm{s})$ con los datos tomados de la tabla 1. Realizando un ajuste de curva polinómica de grado 2 se presenta la expresión matemática con su coeficiente de correlación R. Haciendo un ajuste de los

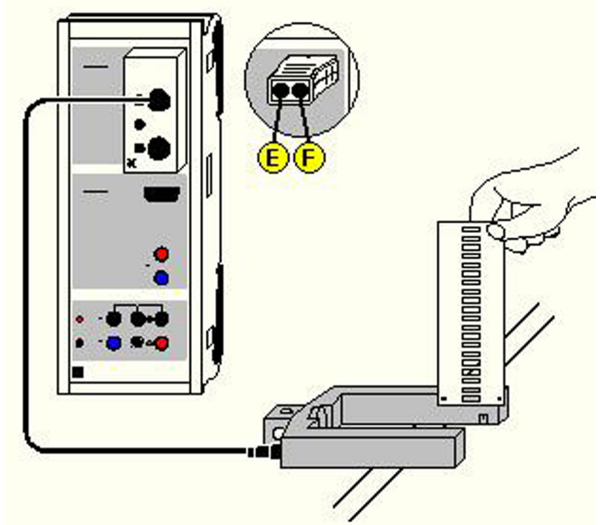

Figura 10: Diagrama del temporizador, sensor CASSY y escalera $\mathrm{g}$ de la marca LEYBOLD [53].

Tabla 1: Tiempos y desplazamientos para una esfera de acero, de radio $6.33 \mathrm{~mm}$., en caída libre medidos con el SAD desarrollado en este trabajo.

\begin{tabular}{ll}
\hline $\mathrm{t}(\mathrm{s})$ & $\mathrm{S}(\mathrm{m})$ \\
\hline 0 & 0 \\
0,080 & 0,04 \\
0,099 & 0,058 \\
0,143 & 0,098 \\
0,144 & 0,115 \\
0,175 & 0,150 \\
0,193 & 0,177 \\
0,214 & 0,247 \\
\hline
\end{tabular}

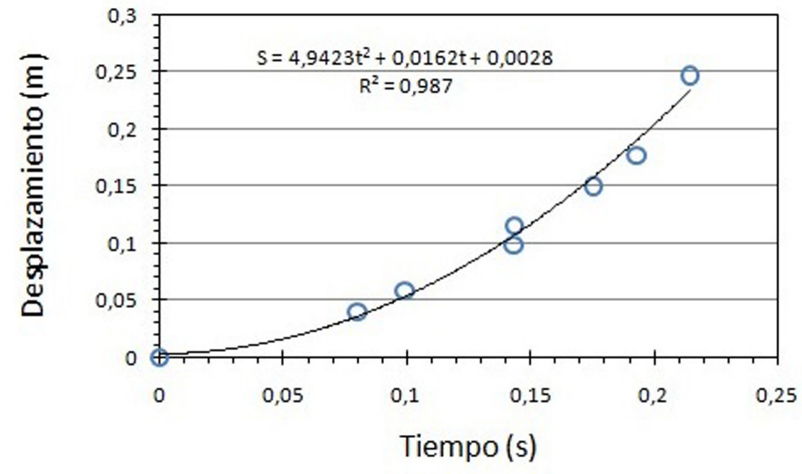

Figura 11: Gráfico de desplazamiento contra tiempo tomando datos con el SAD desarrollado en este trabajo.

datos experimentales medidos con la siguiente ecuación

$$
S=\frac{1}{2} g t^{2}+V_{0} t+S_{0}
$$

se obtiene el siguiente valor para el parámetro g:

$$
g=2(4,9423) \frac{m}{s^{2}}=9,88 \frac{m}{s^{2}},
$$

el cual corresponde al valor central de la aceleración gravitacional en la superficie de la tierra (en Ibagué Colombia, lugar donde se hizo el experimento). Tomando como valor de referencia, la aceleración gravitacional igual a $9,80 \mathrm{~m} / \mathrm{s}^{2}$, el error absoluto es $(9,88-9,80) \mathrm{m} / \mathrm{s}^{2}$ igual a $0,08 \mathrm{~m} / \mathrm{s}^{2}$ y el error relativo es $0,08 / 9,80=8,16 \times 10^{-3}$ que representa un error porcentual de $0.82 \%$.

Los errores estimados en las medidas de la posición y el tiempo son $0.001 \mathrm{~m}$ y $0,0001 \mathrm{seg}$, respectivamente. Considerando a g como una función que depende de la posición y el tiempo, esto es, $\mathrm{g}=\mathrm{g}(\mathrm{s}, \mathrm{t})$, obtenemos

$$
g \pm d g=(9,88 \pm 0,23) \frac{m}{s^{2}} .
$$

El error dg se obtuvo de la expresión

$$
d g^{2}=\left|\frac{\partial g}{\partial s}\right|^{2} d s^{2}+\left|\frac{\partial g}{\partial t}\right|^{2} d t^{2} .
$$

La tabla 2 presenta los tiempos t(s) y los desplazamientos $\mathrm{S}(\mathrm{m})$ para una escalera de aluminio, en caída libre, usando el aparato de la marca LEYBOLD. La figura 12 representa la gráfica del desplazamiento $\mathrm{S}(\mathrm{m})$ contra tiempo $\mathrm{t}(\mathrm{s})$ con estos valores experimentales. Se presenta la expresión matemática con su coeficiente de correlación, haciendo un ajuste de curva polinómica de grado 2. A partir de esta expresión se obtiene que el valor central de la aceleración de la gravedad es $\mathrm{g}=$ $9,68 \mathrm{~m} / \mathrm{s}^{2}$. Comparando con el valor estándar de 9,80 $\mathrm{m} / \mathrm{s}^{2}$ se encuentra que el error absoluto es $(9,80-9.68)$ $\mathrm{m} / \mathrm{s}^{2}$ igual a $0,12 \mathrm{~m} / \mathrm{s}^{2}$ y el error relativo es $0,12 / 9,80=$ $1,2 \times 10^{-2}$ que representa un error porcentual de $1,2 \%$.

En la Tabla 3 se presentan los valores obtenidos, en algunas referencias recientes, para la aceleración de la 
Tabla 2: Tiempos y desplazamientos para una escalera de aluminio, en caída libre, medidos con el instrumento de la marca LEYBLOLD.

\begin{tabular}{ll}
\hline $\mathrm{t}(\mathrm{s})$ & $\mathrm{S}(\mathrm{m})$ \\
\hline 0 & 0 \\
0,018 & 0,01 \\
0,031 & 0,02 \\
0,043 & 0,03 \\
0,054 & 0,04 \\
0,063 & 0,05 \\
0,072 & 0,06 \\
0,080 & 0,07 \\
0,088 & 0,08 \\
0,095 & 0,09 \\
0,102 & 0,1 \\
\hline
\end{tabular}

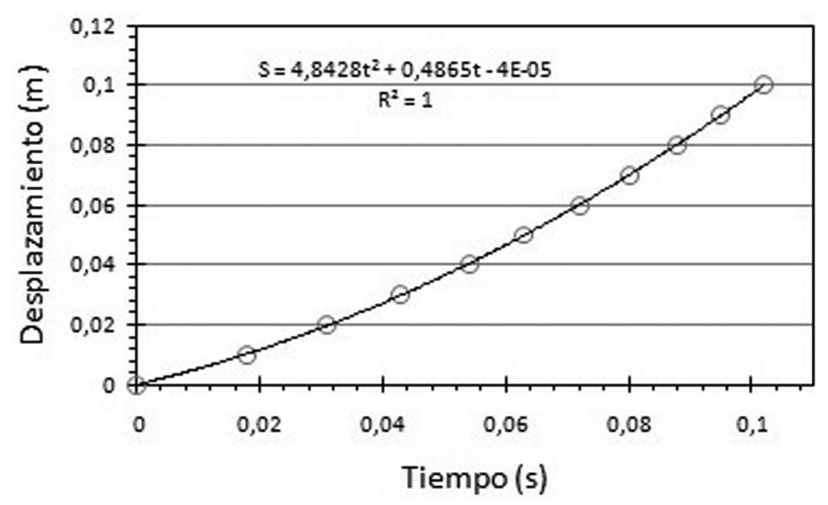

Figura 12: Gráfico de desplazamiento contra tiempo tomando datos con el aparato de caída libre de la marca LEYBOLD.

gravedad (g) usando diferentes dispositivos en distintos experimentos. Las referencias [8], [22], [34] y [45] consideraron un cuerpo en caída libre utilizando el sensor de aceleración de un Smartphone, una placa Arduino, la tarjeta de sonido del computador y una máquina fotográfica digital, respectivamente. En [35] y [37] trabajaron con un cuerpo en movimiento semiparabólico empleando la entrada de micrófono del computador. En el trabajo [42] calcularon el valor de $\mathrm{g}$ a partir del período de un anillo sólido pendular por medio de una fotocompuerta y en [47] a partir del péndulo simple usando un sensor ultrasónico. En la mayoría de estas referencias, el error relativo en el valor de $\mathrm{g}$ se encuentra entre $0,1 \%$ y $1,74 \%$.

Queremos resaltar que el dispositivo desarrollado en el presente trabajo puede ser utilizado en otros experimentos donde se requiera medir la variable tiempo, como por ejemplo: péndulo simple, péndulo físico, masa atada a un resorte, partícula cayendo en un fluido, entre otros.

Por otra parte, la aplicación desarrollada en este trabajo también permite medir la variable física de la temperatura, en experimentos diferentes al de caída libre o experimentos donde se requiera medir tiempo. Es decir, el mismo dispositivo sirve para medir tiempo en unos experimentos o temperatura en otros experimentos. Para esto se requiere configurar algún pin del microcontrolador como análogo.
La opción temperatura del software (ver figura 6) permite dos opciones: una automática que captura cada cierto tiempo la temperatura in situ; y otra, mediante la cual el usuario oprime el botón de captura. El valor de la temperatura se registra en una tabla que contiene las tres escalas más usadas (Celsius, Kelvin y Fahrenheit). Como ilustración, en la figura 13 se muestra la temperatura ambiente medida por nuestro $\mathrm{SAD}$ en la opción automática.

\section{Conclusiones}

En este trabajo se diseñó, construyó e implementó un SAD para medir el tiempo y la temperatura, de bajo costo y de fácil manejo, usando el microcontrolador PIC18F14K50. La programación de éste se realizó con el software gratuito MPLABX y con el compilador XC8. El desarrollo de este tipo de sistemas es una herramienta adicional para el uso de las TIC en la enseñanza de las ciencias naturales, específicamente, en la física. La construcción de este tipo de dispositivos facilita y promueve el desarrollo de la profunda relación que existe entre ciencia básica e ingeniería, como lo es la articulación entre electrónica, programación de sistemas y física, y, contribuye a superar la dificultad de no contar con equipos costosos para realizar experimentos en física en la mayoría de instituciones educativas.

El prototipo desarrollado se utilizó para medir el tiempo en la caída libre de una esfera de acero con el objetivo de obtener, de forma experimental, el valor de la aceleración gravitacional en la superficie de la tierra. A partir de los tiempos y desplazamientos medidos se obtuvo un valor de $g=(9,88 \pm 0,23) \mathrm{m} / \mathrm{s}^{2}$. El error porcentual del valor central respecto al valor de referencia de $9,8 \mathrm{~m} / \mathrm{s}^{2}$ es de $0.82 \%$, el cual es aceptable para un instrumento

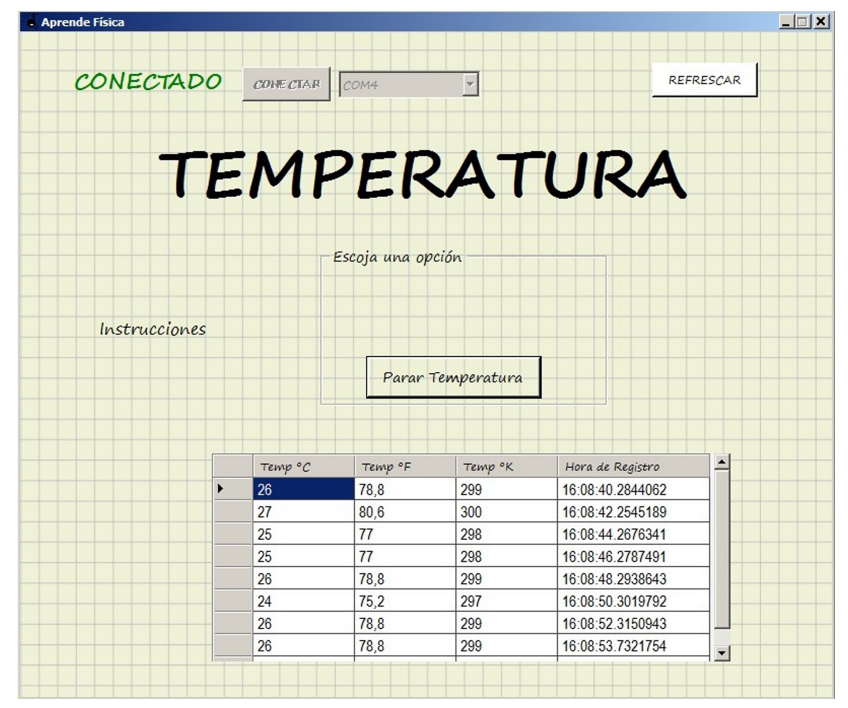

Figura 13: Medición de temperatura ambiente cada 2 ms mediante la opción automática. 
Tabla 3: Valores obtenidos para $\mathrm{g}$ (en $\mathrm{m} / \mathrm{s} 2$ ) usando diferentes dispositivos y distintos experimentos.

\begin{tabular}{cccccccc}
\hline$[8]$ & {$[22]$} & {$[34]$} & {$[45]$} & {$[35]$} & {$[37]$} & {$[42]$} & {$[47]$} \\
\hline $10,0 \pm 0,2$ & $9,78 \pm 0,01$ & 9,780318 & 9,95 & $9,63 \pm 0,55$ & $9,776 \pm 0,005$ & $9,8 \pm 0,2$ & 9,65 \\
\hline
\end{tabular}

de enseñanza básica como el desarrollado aquí en este trabajo.

Un hecho diferenciador del SAD desarrollado en este trabajo es que sirve para medir tiempo o temperatura, de acuerdo al experimento donde se utilice. Así, no se requiere tener dos dispositivos diferentes para medir estas dos variables. Además, su presentación versátil da la posibilidad de ser utilizado en diferentes experimentos de física donde se requiera medir estas dos variables. Por ejemplo, el dispositivo se puede utilizar para medir el período de un péndulo al disponer la torre de la figura 5 en forma horizontal; de igual forma si se coloca formando un plano inclinado con la horizontal, se puede utilizar para estudiar movimiento uniforme o uniformemente acelerado.

\section{Referencias}

[1] S.E. Calderón, P. Núñez, J.L. Di Laccio, L.M. Iannelli y S. Gil, Revista Eureka sobre Enseñanza y Divulgación de la Ciencias 12, 212 (2015).

[2] A. Pontes, J. Gavilán, M. Obrero y A. Flores, Revista Eureka sobre Enseñanza y Divulgación de las Ciencias 3, 251 (2006).

[3] V.C. Capuano, Virtualidad, Educación y Ciencia 2, 79 (2011).

[4] S. López, E.A. Veit e I. Solano Araujo, Revista Brasileira de Ensino de Física 38, 2401 (2016).

[5] M.E. Cardona y S. López, Revista Brasileira de Ensino de Física 39, 4404 (2017).

[6] S. Gil y J.L. Di Laccio, Lat. Am. J. Phys. Educ. 11, 1305 (2017).

[7] J. Molina-Coronell, W. Celin Mancera y C. Solano Mazo, Revista Mexicana de Física E 63, 76 (2017).

[8] J. Kuhn and P. Vogt, Eur. J. Phys. Edu. 4, 16 (2013).

[9] P. Vogt and J. Kuhn, The Physics Teacher 50, 182 (2012); The Physics Teacher 51, 118 (2013); The Physics Teacher 51, 182 (2013); Frontiers in Sensors 1, 67 (2013); The Physics Teacher 52, 118 (2014).

[10] M. Monteiro, C. Stari, C. Cabeza and A.C. Marti, The Physics Teacher 53, 373 (2015).

[11] M. Monteiro, C. Cabeza e A.C. Marti, Revista Brasileira de Ensino de Física 37, 1303 (2015).

[12] J. Kuhn, Am. J. Phys. 82, 94 (2014).

[13] http://www.phonelabs.net/, acceso en 20/05/2017.

[14] http://physlets.org/tracker/, acceso en 23/05/2017.

[15] C. Sirisathitkul, P. Glawtanong, T. Eadkong e Y. Sirisathitkul, Revista Brasileira de Ensino de Física 35, 1504 (2013).

[16] A.C. Wrasse, L. Patron, G.F. Marranghello e F. Saraiva da Rocha, Revista Brasileira de Ensino de Física 36, 1501 (2014).

[17] V.L.B. de Jesus e D.G.G. Sasaki, Revista Brasileira de Ensino de Física 36, 3503 (2014); Revista Brasileira de Ensino de Física 37, 1507 (2015).
[18] W. Bonventi e N. Aranha, Revista Brasileira de Ensino de Física 37, 2504 (2015).

[19] https://phet.colorado.edu/, acceso en 26/05/2017.

[20] G. Fetzner Filho, Experimentos de Baixo Custo Para o Ensino de Física em Nivel Médio Usando a Placa Arduino-UNO. Mestrado Profissional em Ensino de Física, Universidade Federal do Rio Grande do Sul, Porto Alegre (2015).

[21] R. Frank de Rodrigues e S.L. Souza Cunha, Textos de Apoio ao Profesor de Física 25, n. 4 (2014).

[22] H. Cordova e A.C. Tort, Revista Brasileira de Ensino de Física 38, 2308 (2016).

[23] H. Salim do Amorim, M.A. Dias e V. Soares, Revista Brasileira de Ensino de Física 37, 4310 (2015).

[24] M. Varanis, A. Langone, P.H. Ayres e R. Ferreira, Revista Brasileira de Ensino de Física 38, 1301 (2016).

[25] M.A. Cavalcante, C.R.C. Tavolaro e E. Molisani, Revista Brasileira de Ensino de Física 33, 4503 (2011).

[26] C.T.W. da Rosa, M.A. Trentin, A.B. da Rosa e A.C. Giacomelli, Caderno Brasileiro de Ensino de Física 33, 292 (2016); A.A.M. Santos, H.S. Amorim e C.P. Dereczynski, Revista Brasileira de Ensino de Física, 39, 1505 (2017); B. Huang, Open-source Hardware - Microcontrollers and Physics Education - Integrating DIY Sensors and Data Acquisition with Arduino, 122nd ASEE Anual Conference \& Exposition, June 14-17, Seattle, 2015; J. Rosenberg and K. Cuff, Lat. Am. J. Phys. Educ. 6, Suppl. I, 39 (2012).

[27] A.R. de Souza, A.C. Paixao, D.D. Uzeda, M.A. Dias, S. Duarte e H.S. de Amorim, Revista Brasileira de Ensino de Física 33, 1702 (2011).

[28] V.R. Coluci, G. Paulino, D.C. de Souza e E.P.R. Vasconcelos, Revista Brasileira de Ensino de Física 35, 2506 (2013).

[29] L.H.M. de Castro, B.L. Lago e F. Mondaini, Journal of Applied Mathematics and Physics 3, 631 (2015).

[30] C. Galeriu, The Physics Teacher 51, 156 (2013).

[31] J.C. Andrades, A. Schiappacassa e P. Freire dos Santos, Revista Brasileira de Ensino de Física 35, 2503 (2013).

[32] H.A. Gallego, H. Orozco y C.A Cortés, Revista Scientia et Technica 37, 539 (2007).

[33] W. Celin Mancera, C. Solano Mazo y J. Molina Coronell, Revista Espacios 38, 9 (2017).

[34] R.G. Camero-Berrones, M. Zapata-Torres, O. CalzadillaAmaya, J. Guillen Rodríguez y J. Laria- Menchaca, Revista Mexicana de Física E 58, 18 (2012).

[35] M.A. Cavalcante, A. Bonizzia e L.C. Pereira Gomes, Revista Brasileira de Ensino de Física 31, 4501 (2009).

[36] J.S. Figueira e E.A. Veit, Revista Brasileira de Ensino de Física 26, 203 (2004).

[37] J.A. White, A. Medina, F.L. Román and S. Velasco, The Physics Teacher 45, 175 (2007).

[38] F.F. Luiz, L.E.S. Souza e P.H. Domingues, Revista Brasileira de Ensino de Física 38, 2504 (2016).

[39] S.E. Calderón, P. Nuñez y S. Gil, Lat. Am. J. Phys. Educ. 4, 188 (2010). 
[40] E. de Barros, C. d'Andrade, L. Ribeiro de Camargo e M.H. Mathias, Revista Brasileira de Ensino de Física 37, 4313 (2015).

[41] G. Dionisio e W.C. Magno, Revista Brasileira de Ensino de Física 29, 287 (2007).

[42] R. De Luca e S. Ganci, Revista Brasileira de Ensino de Física 33, 3301 (2011).

[43] K. Madhur, P. Arun and F.M.S. Lima, arXiv:physics/ 0604106v1 [physics.ed-ph].

[44] A. Wooton, The Physics Teacher 38, 405 (2000).

[45] E.P. Moraes Corveloni, E.S. Gomes, A.R. Sampaio, A.F. Mendes, V.L.L. Costa e R.C. Viscovini, Revista Brasileira de Ensino de Física 31, 3504 (2009).

[46] M.A. Dias, H.S. de Amorim e S. de Souza Barros, Cad. Bras. Ens. Fis. 26, 492 (2009).

[47] F.J. Arnold, R. Arthur, L.L. Bravo-Roger, M.S. Goncalves e M.J. Garrido de Oliveira, Revista Brasileira de Ensino de Física 33, 4311 (2011).

[48] http://www.microchip.com, acceso en 05/01/2017.

[49] http://www.microchip.com/mplab/mplab-x-ide, acceso en $05 / 01 / 2017$.

[50] http://ww1.microchip.com/downloads/en/ DeviceDoc/41350C.pdf acceso en 05/01/2017.

[51] http://www.ti.com/, acceso en 20/01/2017.

[52] M. Verle, PIC Microcontrollers Programming in Basic (MikroElektronika, USA, 2009), disponible en https://learn.mikroe.com/ebooks/ picbasicprogramming/front-matter/introduction/, acceso en $05 / 01 / 2017$.

[53] www.ld-didactic.de/software/524221es/Content/ ExperimentExamples/Physics/Mechanics/FreeFall. htm, acceso en 18/04/2017. 